\title{
CHAOTIC DYNAMICS
}

\author{
J. PALIS \\ Instituto de Matemática Pura e Aplicada, Estrada Dona Castorina 110, \\ Jd. Botânico, 22.460 - Rio de Janeiro, Brazil
}

Two important theories in Dynamical Systems were constructed in the sixties: the hyperbolic theory for general systems and the KAM (after Kolmogorov, Arnold and Moser) theory for conservative systems as the ones that appear in Celestial Mechanics. Most of our discussions here concern dissipative (or locally dissipative) systems, although most questions are now being posed for area preserving maps (symplectic maps in higher dimensions). Moreover, one can argue that understanding dynamically small dissipative perturbations of conservative systems is of much importance: indeed it has been recently shown that a KAM curve (tori in higher dimension) can be destroyed and in fact engulfed in the basin of attraction of a Hénon-like strange attractor as defined below.

Let me say that all concepts, many proofs and more detailed discussions of the topics in the present paper are about to appear in a book by Takens and myself (Palis and Takens, 1992).

Recall that a hyperbolic system (flow, diffeomorphism, map) is one whose limit set is hyperbolic. That is, in the limit set, all Lyapunov exponents are uniformly bounded away from zero. In the case of flows we exclude the one along the flow, which is of course always zero, when stating this exponential growth condition. In our presentation, we shall concentrate our attention to maps, more properly to diffeomorphisms of a compact manifold. They may be consider as Poincaré transformations of a flow in one higher dimension. They are indicated by $\operatorname{Diff}^{\mathrm{r}}(\mathbf{M}), r \geq 1$, where $M$ is a compact manifold and $r$ the degree of differentiability considered: two such maps are close if pointwise they are close as well as all their derivatives are close up to order $r$ ( $C^{r}$ topology). For our purposes, one may take $r=\infty$.

Much has been done about hyperbolic systems (including most gradient systems) in the sixties, its leading figure being Smale (his school at the time and the Russian school played crucial roles). Indeed we "pretend" that we understand them rather well. Actually, we were so enthusiastic about it that in some sense we though that a large part of the "world" of dynamical systems was made of hyperbolic systems. A great change in our view began to take place in the seventies due to the work, mostly based on computational experiments, of physicists, astronomers and all (officially nonmathematicians) such as Lorenz (his remarkable work was only noticed by us in the early seventies), Hénon, Ruelle, Feigenbaum. The drama that turned into a big challenge to us, dynamicists, was: most of the computational experiments corresponding to dynamical models of complex phenomena in other areas of Science did not seem to fit the hyperbolic structure. Rather, they seemed to lie in the complement of the hyperbolic systems, the dark realm of the world of dynamics. The figures display two very famous strange attractors (Lorenz, Hénon) and a new one discovered by Rovella, all of them robust in some sense, as discussed below, and yet all of them nonhyperbolic! 


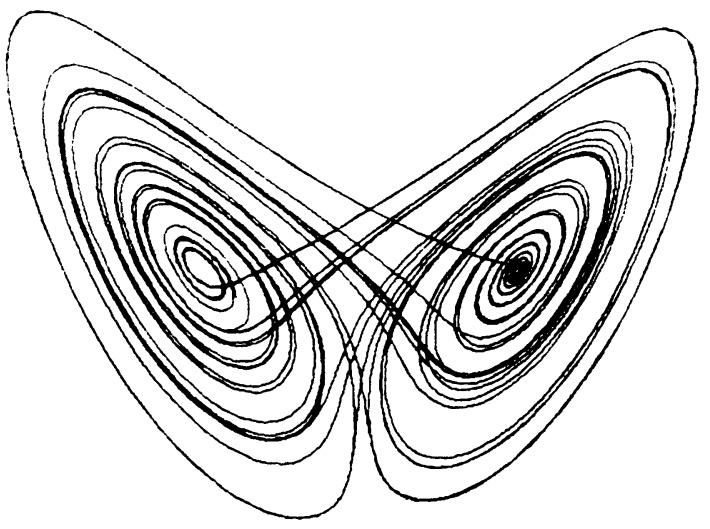

Fig. 1. Lorenz strange attractor

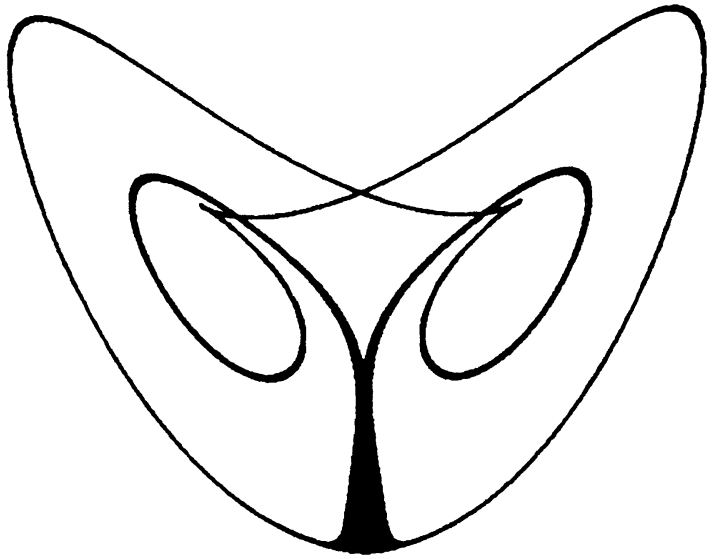

Fig. 2. Rovella strange attractor

This led me, in the last seven years or so, to intensely discuss a new viewpoint or a global program for (locally dissipative) dynamical systems with several colleagues, specially Takens, Yoccoz, Viana, Newhouse, Mañé, Carleson, Mora, Rovella, Carvalho, Diaz and Rocha. A series of results motivated the program I present here and perhaps some of these results were at least partially motivated by it.

The basic idea is to define a dense subset $\mathcal{H}$ of all dynamical systems, say $\mathcal{H} \subset \operatorname{Diff}^{\mathrm{r}}(\mathrm{M})$, and then describe the prevalent dynamical phenomena in small neighbourhoods of the element of $\mathcal{H}$ in generic or most $k$-parameter families of diffeomorphisms through them.

We should of course first consider $M$ to be compact without boundary and ideally $r$ should be bigger than two. 


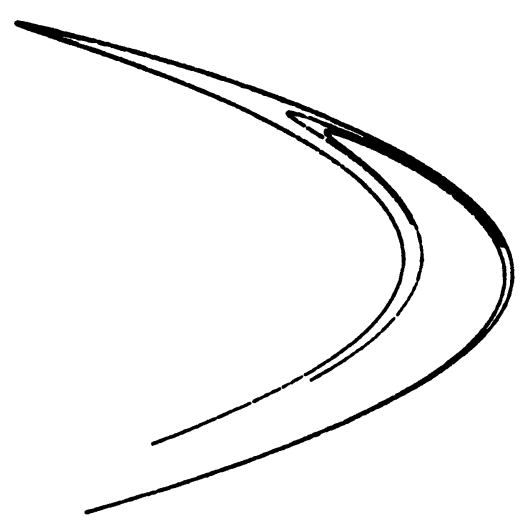

Fig. 3. Hénon strange attractor

If $\operatorname{dim} M=2$, we think that a good candidate for $\mathcal{H}$ is the union of hyperbolic diffeomorphisms and the ones exhibiting homoclinic tangencies. In higher dimension, as we shall discuss in the sequel, we need to consider homoclinic bifurcations in more generality than homoclinic tangencies. One may add the so called Feigenbaum attractors (see Gambaudo et al., 1989) and, as we shall discuss later, diffeomorphisms with a critical saddle-node cycle. Anyhow, $\mathcal{H}$ should be formed by hyperbolic diffeomorphisms, that we "pretend" to understand rather well, together with a set of diffeomorphisms that, on one hand, is dense in the complement and, at the same time, is not "too big" so as to allow a reasonable dynamic analysis in small neighbourhoods of its elements in finite dimensional spaces through them.

Conjecture. $\mathcal{H}$ as above is dense in $\operatorname{Diff}^{\mathrm{r}}(\mathrm{M}), r \geq 1$.

One reason to consider surface diffeomorphisms with homoclinic tangencies is the striking dynamic richness of their one-parameter unfoldings. So let $\varphi_{\mu}, \mu \in \mathbf{R}$, be a family of $C^{3}$ diffeomorphisms such that $\varphi_{0}$ exhibits a homoclinic tangency say $q_{0}$ associated to a fixed (periodic) saddle $p_{0}$. We assume that the product of the eigenvalues of $(d \varphi)_{0}$ has norm smaller than one (i.e. $\varphi_{0}$ is locally dissipative). The saddle $p_{0}$ may be part of a (hyperbolic) basic set say $\Lambda_{0}$ (otherwise we take $\left.\Lambda_{0}=p_{0}\right)$. We now localize our study to a $\mu$-dependent neighbourhood $U_{\mu}$ of the orbit of $q_{0}$ and of $\Lambda_{0}$. First, we define a fixed neighbourhood $U_{1}$ of $\Lambda_{0}$ and $\varepsilon>0$ small so that, for $|\mu|<\varepsilon$, there is a (unique) basic set $\Lambda_{\mu}$ in $U_{1} ; \Lambda_{\mu}$ is called the continuation of $\Lambda_{0}$. Now we define a $\mu$-dependent neighbourhood $U_{2, \mu}^{*}$ of $q_{0}$ whose points are at a distance at most $K \mu$, for some constant $K>0$, from both the local stable and unstable manifold of $p_{\mu}$. Finally, we consider $N>0$ so that

$$
U_{\mu}=U_{1} \cup U_{2, \mu}
$$

is a neighbourhood of $q_{0}$ and $\Lambda_{0}$, where

$$
U_{2, \mu}=\bigcup_{|i| \leq N} \varphi_{\mu}^{i}\left(U_{2, \mu}^{*}\right)
$$


We then have the following collection of results concerning $\varphi_{\mu} \mid U_{\mu}$ :

- there are always cascades of period doubling bifurcations in the family $\varphi_{\mu}$ for $\mu \rightarrow 0$ (Yorke and Alligood, 1983),

- there are intervals $I_{i} \subset(-\varepsilon, \varepsilon), \varepsilon$ small, and residual subsets $N_{i} \subset I_{i}$ such that $\varphi_{\mu}$, for $\mu \in N_{i}$, exhibits infinitely many coexisting sinks (Newhouse, 1979 ),

- there are positive Lebesgue measure sets $E \subset(-\varepsilon, \varepsilon)$ such that if $\mu \in E$ then $\varphi_{\mu}$ has a Hénon-like attractor (Mora and Viana, 1991 based on Benedicks and Carleson, 1991),

- if the Hausdorff dimension of $\Lambda_{0}, H D\left(\Lambda_{0}\right)$, is smaller than one, then $\mu=0$ is a (full) density point of hyperbolicity for $\varphi_{\mu}$ (Palis and Takens, 1987),

- if $H D\left(\Lambda_{0}\right)>1$, then $\mu=0$ is not a point of (full) density of hyperbolicity for $\varphi_{\mu}$ (Palis and Yoccoz, 1991).

It is interesting to note that in Araujo and Mañé (1991) our conjecture above was proved in the $C^{1}$ topology for $C^{2}$ diffeomorphisms in the following "essential" way: residually a diffeomorphism has the basins of attraction of its hyperbolic attractors covering a set of total measure (i.e. covering the surface modulo a set of Lebesgue measure zero) or else the diffeomorphism exhibits a homoclinic tangency.

Concerning families $\varphi_{\mu}$ exhibiting a critical saddle-node cycle say at $\mu=0$, there is a recent relevant result of Diaz, Rocha and Viana (1991) stating that $\mu=0$ is a positive density point of Hénon-like strange attractors. It is to be noted that such critical saddle-node cycles yield homoclinic tangencies for $\varphi_{\mu},|\mu|$ small (Newhouse et al.1983). A clever combination of this fact and the previously mentioned result of Mora and Viana provides the present result. So far, this is the only known dynamic structure corresponding to positive density of parameter values corresponding to diffeomorphisms having Hénon-like attractors for generic one-parameter families of surface diffeomorphisms, i.e. nonhyperbolic attractors that are the closure of the unstable manifold of a saddle and, have dense orbit with positive Lyapunov exponent. The Diaz-Rocha-Viana method to prove the above result also yields positive density of parameter values corresponding to diffeomorphisms exhibiting sinks.

Problem. Let $\varphi_{\mu}, \mu \in \mathbf{R}$, be a (generic) family of surface diffeomorphisms such that

- $\varphi_{\mu}$ is Morse-Smale for $\mu<0$

- the limit set of $\varphi_{0}$ is finite, hyperbolic and has no cycle except for a critical saddle-node cycle.

Is it true (in increasing order of difficulty) that:

- the set of parameter values corresponding to hyperbolic diffeomorphisms is of positive density at $\mu=0$ ?

- the set of parameter values corresponding to diffeomorphisms exhibiting infinitely many coexisting sinks is of zero density at $\mu=0$ ?

- the union of the sets of parameter values corresponding to hyperbolic diffeomorphisms and to the ones exhibiting a Hénon-like strange attractor is of full density at $\mu=0$ ? 
In general, we pose

Conjecture. In a (generic) unfolding of a homoclinic tangency or a saddle-node critical cycle, the set of parameter values corresponding to infinitely many coexisting sinks has Lebesgue measure zero.

$A$ weaker version of this conjecture is to ask if the bifurcating value is a zero density point of the set of parameter values corresponding to diffeomorphisms exhibiting infinitely many sinks. Notice that the answer is positive when $H D\left(\Lambda_{0}\right)<1$ in the case of a homoclinic tangency by the result in Palis and Takens (1987).

Besides the result in Palis and Yoccoz (1991) stating that hyperbolicity is not fully prevalent in the unfolding of a homoclinic tangency when $H D\left(\Lambda_{0}\right)>1$, we recall that if $\tau^{\prime}\left(\Lambda_{0}\right) \cdot \tau^{u}\left(\Lambda_{0}\right)>1$, where $\tau^{\prime}$ and $\tau^{u}$ stands for stable and unstable thickness, then we have persistence of tangencies for $|\mu|$ small and $\mu>0$ or $\mu<0$ and thus we have no hyperbolicity at all in this range of the parameter. In both these situations (the second, $\tau^{s} \cdot \tau^{u}>1$, is a particularly interesting case of the first, $H D\left(\Lambda_{0}\right)>1$ ) the following is a very interesting problem:

Problem. Is the bifurcating value (unfolding of a homoclinic tangency) of positive density of Hénon-like strange attractor?

We point out that in higher dimensions we may have homoclinic bifurcations without homoclinic tangencies! Consider for instance two fixed saddles $p$ and $q$, for a diffeomorphism $\varphi$ in 3-dimensions with stable indices one and two, respectively. Suppose moreover that $W^{u}(p)$ intersects transversally $W^{s}(q)$ along a line connecting $p$ and $q$. Finally, suppose that $W^{u}(q)$ intersects $W^{*}(p)$. According to the results in Diaz (1991), there are open sets of diffeomorphisms that are neither hyperbolic nor exhibit homoclinic tangencies after a generic 1-parameter unfolding of $\varphi$, if $\varphi$ is suitably chosen. Similarly, one may consider the global examples in Mañé (1978). Thus our first conjecture for surface diffeomorphisms become

Conjecture. The set of hyperbolic diffeomorphisms together with the ones exhibiting homoclinic bifurcations is dense in $\operatorname{Diff}^{\mathrm{r}}(\mathrm{M}), r \geq 1$.

Also, some of the previous results concerning unfolding of homoclinic tangencies are now available in higher dimensions:

- if $H D\left(\Lambda_{0}\right)<1$, hyperbolicity is fully prevalent, ,

- there are cascades of period doubling bifurcations; this is in Yorke and Alligood (1983) when the unstable index is one (codimension-one case) and, in general, in a recent work of C. Martins,

- when the unstable index is one, there are intervals $I_{i}$ in the parameter line and residual subsets $N_{i} \subset I_{i}$ such that for parameter values in $N_{i}$ the corresponding diffeomorphisms exhibit infinitely many coexisting sinks (Palis and Viana, 1991),

- again, when the unstable index is one, there are positive Lebesgue measure sets in the parameter line whose corresponding diffeomorphisms exhibit Hénon-like attractors (Viana, 1991).

Finally, the scenario for flows is even more challenging: besides the "suspension" of the corresponding cases of diffeomorphisms, we have to add homoclinic bifurcations of singularities (Silnikov, 1965), the Lorenz-like attractors (Lorenz, 1963, 
Guckenheimer and Williams, 1979, Williams, 1979, Rovella, 1991) and the very interesting singular horseshoes (Labarca and Pacífico, 1986, Bamón et al., 1991). I hope that in the near future more results will be available in that direction and through them and what we already know, a richer but similar global program to the one we have presented diffeomorphisms can be also proposed for flows.

\section{References}

Araujo, A. and Mañé, R.: 1991 "On the existence of hyperbolic attractors and homoclinic tangencies for surface diffeomorphisms", to appear.

Benedicks, M. and Carleson, L.: 1991 "The dynamics of the Hénon map", Annals of Math. 133, 73-169.

Bamón, R., Labarca, R., Mañé, R. and Pacífico, M.J.: 1991, "Bifurcating 3-dimensional singular cycle", to appear.

Diaz, L. "Persistence of nonhyperbolicity and heterodimensional cycles", Thesis, IMPA, and to appear.

Diaz, L. Rocha, R. and Viana, M.: 1991, "Saddle-node critical cycles and prevalence of strange attractors", to appear.

Gambaudo, J.-M., von Strein, S. and Tresser, C.: 1989 "Hénon-like maps with strange attractors: there exist $C^{\infty}$ Kupka-Smale diffeomorphisms on $S^{2}$ with neither sinks or sources", Nonlinearity 2, 287-304.

Guckenheimer, J. and Williams, R.F.:1979 "Structural stability of Lorenz attractors", Publ. Math. I.H.E.S. 50, 59-72.

Lorenz, E. N.: 1963 "Deterministic non-periodic flow", J.Atmos. Sci. 20, 130-141.

Labarca, R. and Pací fico, M.J.: 1986 "Stability of singular horseshoe", Topology 25, 337-352.

Mañé, R."Contribution to the stability conjecture", Topology 17, 383-396.

Mora, L. and Viana, M.: 1991 "Abundance of strange attractors", Acta Math., to appear.

Newhouse, S.: 1979 "The abundance of wild hyperbolic sets and nonsmooth stable sets for diffeomorphisms", Publ. Math. I.H.E.S. 50, 101-151.

Newhouse, S., Palis, J. and Takens, F.: 1983 "Bifurcations and stability of families of diffeomorphisms", Publ. Math. I.H.E.S. 57, 5-71.

Palis, J. and Takens, F.: 1987 "Hyperbolicity and the creation of homoclinic orbits", Annals of Math. 125, 337-374.

Palis, J. and Takens, F.: 1992 Hyperbolicity and sensitive chaotic dynamics at homoclinic bifurcations, Cambridge University Press.

Palis, J. and Viana, M:1991 "Infinitely many sinks in higher dimensions", to appear.

Palis, J. and Yoccoz, J.C.: 1989 "Homoclinic tangencies for hyperbolic sets of large Hausdorff dimension", to appear.

Robinson, C.: 1989 "Homoclinic bifurcation to a transitive attractor of Lorenz type", Nonlinearity 2, 495-518.

Rovella, A.: 1991 "The dynamics of the perturbations of the contracting Lorenz attractor", Thesis, IMPA, and to appear.

Rychlik, M.:1990 "Lorenz's attractors through Silnikov-type bifurcation", Erg. Th. and Dyn. Syst. 10, 793-821.

Silnikov, L.P.:1965 "A case of the existence of denumerable set of periodic motions", Sov. Math. Dokl. 6, 163-166.

Takens, F.: 1991 "On the geometry of non-transversal intersections of invariant manifolds and scaling properties of bifurcation sets", Pitman Research Notes in Math. Series, to appear.

Takens, F.: 1986 "Homoclinic bifurcations" Proc. Int. Congress of Math., Berkeley, 1229-1236.

Viana, M.: 1991 "Strange attractors in higher dimensions, Thesis, IMPA, and to appear.

Williams, R.F.: 1979 "The structure of Lorenz attractors", Publ. Math. I.H.E.S. 50, 101-152.

Yorke, J.A. and Alligood, K. T.: 1983 "Cascades of period doubling bifurcations: a prerequisite for horseshoes", Bull. A.M.S. 9, 319-322. 